\title{
Optimizing care for psoriatic patients requiring systemic therapies: how will COVID-19 disease transform risk perceptions?
}

\author{
M. Talamonti ${ }^{1,2} \odot \cdot$ L. Tofani ${ }^{1,2} \cdot$ L. Bianchi ${ }^{1,2} \cdot$ M. Galluzzo $0^{1,3}$
}

Received: 22 May 2020 / Revised: 5 June 2020 / Accepted: 30 June 2020 / Published online: 9 July 2020

(c) Springer-Verlag GmbH Germany, part of Springer Nature 2020

\section{Dear Editors,}

Many authors have recently published recommendations for patient treatment during the Coronavirus disease 2019 (COVID-19) pandemic [1]. Coronavirus disease 2019 (COVID-19) pandemic outcomes seem to be determined by the extent of imbalances in the host immune system [2]. The primary immune response is positive and leads to viral clearance in the majority of cases. However, for reasons that are unclear, the secondary immune response ("cytokine storm") may be exaggerated and challenge tissue integrity, sometimes leading to multiple organ failure, acute respiratory distress syndrome (ARDS), and death [2] (Fig. 1). As of 18 May 2020, Italy had 225,549 confirmed COVID-19 cases and 30,332 associated deaths. The median age of patients dying was 80 years; $60.7 \%$ of these presented with 3 or more comorbidities (Table 1) [3]. In accordance with recommendations from dermatology societies [4], patients under treatment including biologic agents and apremilast, and/or immunosuppressants were advised not to discontinue their drugs without consultation; cessation of treatment was suggested only with signs of infection. We agree with many suggestions that the interruption of therapies may cause the dysregulation of inflammatory cytokines that may not only exacerbate the disease itself, but may also be involved in the pathogenesis of the viral infection. Actually, there is not an agreement nor a study sustaining the impact of continuing

M. Talamonti, L. Tofani, L. Bianchi and M. Galluzzo have contributed equally to this work.

M. Talamonti

talamonti.marina@gmail.com

1 Dermatology Unit, Fondazione Policlinico Tor Vergata, Rome, Italy

2 Department of Systems Medicine, University of Rome "Tor Vergata", Rome, Italy

3 Department of Experimental Medicine, University of Rome "Tor Vergata", Rome, Italy or stopping treatments in psoriatic patients during the COVID-19 pandemic [4]. But the issue of starting any systemic treatment now or in the coming weeks has not yet been addressed. Immunosuppressants (i.e., corticosteroids, methotrexate, cyclosporine) are associated with an increased risk of infection. The risk is usually dose dependent, varies with each agent, and often relates more to the underlying health condition being treated. Clinical trials and real evidence on biologics (i.e., TNF- $\alpha$, IL-17, IL-23, and IL12/-23 inhibitors) do not show substantial increases in infection risk compared to placebo [5]. Until further evidence is available, the risks and benefits of initiating systemic therapy should be examined on an individual basis, considering the risk of exposure to COVID-19 based on occupation or housing situation and the following factors: endemic area, jobs requiring frequent/close contact with people who may be infected but are not known or suspected patients, healthcare workers, infected family members or co-workers, nursing home residents. In addition, we advise caution starting an immunosuppressive therapy in the presence of risk factors for COVID-19 mortality such as age $>60$, hypertension, diabetes and obesity, which are common in psoriatic patients (Table 1). Another logistical parameter that should not be underestimated is the need for frequent careful monitoring during immunosuppressants, with laboratory examinations [5] and routine dermatological follow-ups, which could be problematic under the restrictions on movement. Moreover, now more than ever, biological therapies should be chosen as safer therapeutic options that decrease the rate of morbidity and the risks connected to immunosuppressive therapies. We have highlighted an issue about the drugs chosen by patients who are candidates for systemic therapies in the era of COVID-19. Given all of the above, the authors' personal opinion is that only biologic treatments or apremilast should be considered when possible in this particular period. 
Median age (years)

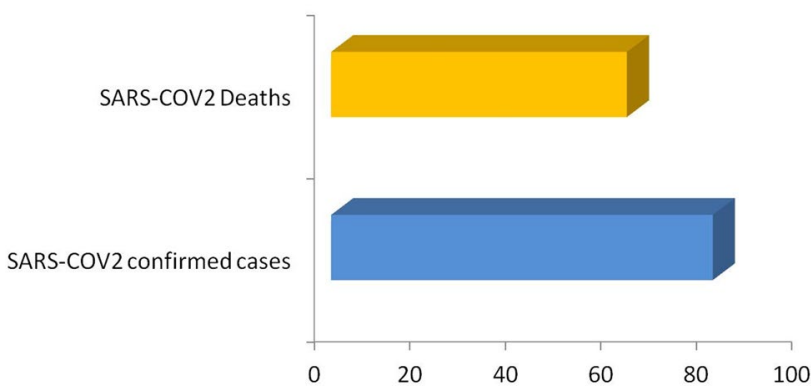

Fig. 1 Median age of patients with SARS-CoV-2 infection and SARS-CoV-2-positive deceased patients

Table 1 Most common comorbidities observed in SARS-CoV-2-positive deceased patients

\begin{tabular}{lrl}
\hline Diseases & $N$ & $\%$ \\
\hline Hypertension & 1317 & 69.7 \\
Type 2 diabetes & 603 & 31.9 \\
Ischemic heart disease & 518 & 27.4 \\
Atrial fibrillation & 411 & 21.7 \\
Chronic renal failure & 405 & 21.4 \\
COPD (chronic obstructive pulmonary & 327 & 17.3 \\
$\quad$ disease) & & \\
Active cancer in the past 5 years & 301 & 15.9 \\
Heart failure & 298 & 15.8 \\
Dementia & 280 & 14.8 \\
Obesity & 230 & 12.2 \\
Stroke & 206 & 10.9 \\
Number of comorbidities & & \\
1 comorbidity & 273 & 14.4 \\
2 comorbidities & 400 & 21.2 \\
$\geq 3$ comorbidities & 1147 & 60.7 \\
\hline
\end{tabular}

\section{Compliance with ethical standards}

Conflict of interest None of the authors have conflicts of interest to disclose.

\section{References}

1. Zhao M (2020) Cytokine storm and immunomodulatory therapy in COVID-19: role of chloroquine and anti-IL-6 monoclonal antibodies. Int J Antimicrob Agents 16:105982. https://doi. org/10.1016/j.ijantimicag.2020.1059824

2. Epicentro ISS. Epidemia COVID-19, available via https://www. epicentro.iss.it/

3. European Academy of Dermatology and Venereology (EADV). https://eadv.org/covid-19/task-force

4. Lebwohl M, Rivera-Oyola R, Murrell DF (2020) Should biologics for psoriasis be interrupted in the era of COVID-19? J Am Acad Dermatol. https://doi.org/10.1016/j.jaad.2020.03.031

5. Gisondi P, Altomare G, Ayala F et al (2017) Italian guidelines on the systemic treatments of moderate-to-severe plaque psoriasis. J Eur Acad Dermatol Venereol 31:774-790. https://doi. org/10.1111/jdv. 14114

Publisher's Note Springer Nature remains neutral with regard to jurisdictional claims in published maps and institutional affiliations. 